\title{
Cambios en la morfometría bucal y su relación con la dieta de Thyrsites atun (Euphrasen, 1791) en el centro-sur de Chile
}

\author{
Changes in mouth morphometrics as related to the diet of Thyrsites atun (Euphrasen, 1791) \\ from south-central Chile
}

FREDDY DUARTE ${ }^{1}$, CHRISTIAN M. IBÁÑEZ $^{2 *} \&$ JAVIER CHONG $^{1}$

\author{
${ }^{1}$ Departamento de Ecología Costera, Facultad de Ciencias, Universidad Católica de la Santísima Concepción, \\ Casilla 297, Concepción, Chile \\ ${ }^{2}$ Instituto de Ecología y Biodiversidad, Departamento de Ciencias Ecológicas, Facultad de Ciencias, Universidad de Chile, \\ Casilla 653, Ñuñoa, Santiago, Chile; \\ *e-mail for correspondence: christianibez@yahoo.com
}

\begin{abstract}
RESUMEN
Varias especies de peces carnívoros, a medida que crecen, tanto el tamaño corporal como el de la boca, se relacionan positivamente con el tamaño de las presas. Sin embrago, el número y la biomasa de las presas consumidas por los peces no presentan un patrón tan claro. Para verificar estas relaciones en el pez Thyrsites atun, se recolectaron entre los meses de febrero y abril de 2004, un total de 140 ejemplares, desde desembarques de naves pesqueras industriales y semiindustriales en la región centro-sur de Chile. Se estableció la dieta de T. atun mediante descriptores cuantitativos de frecuencia, número y peso de las presas, los cuales se compararon entre peces subadultos y adultos. También registramos medidas morfométricas bucales para compararlas con el tamaño del pez y de las presas, y también se estimó el volumen de la boca para relacionarlo con el número y peso de las presas ingeridas. En sus tractos digestivos se encontraron cuatro especies de presas, Euphausia sp., Todarodes filippovae, Strangomera bentincki y Engraulis ringens, siendo esta última la presa más frecuente $(99,9 \%)$. Las medidas bucales resultaron dependientes del tamaño del depredador. Igualmente el tamaño de las presas resultó ser dependiente del tamaño del depredador, así como de las medidas bucales. Además, el volumen de la boca se relacionó positivamente con la longitud del depredador, y tanto el número como el peso de las presas se correlacionaron con el tamaño y volumen de la boca. Thyrsites atun se alimenta en la zona nerítica de peces de pequeño tamaño que viven en cardúmenes. Al aumentar de tamaño $T$. atun se alimentaría de presas más grandes, y consecuentemente al incrementarse el volumen de la boca puede comer una mayor cantidad y biomasa de presas.
\end{abstract}

Palabras clave: Thyrsites atun, morfometría bucal, tamaño corporal, alimentación, cardúmenes de peces.

\begin{abstract}
In several species of carnivorous fishes, as they grow, body size and that of the mouth are related positively with prey size, however, the number and biomass of preys consumed by the fish don't present such a clear pattern. To verify these relationships in the fish Thyrsites atun, a total of 140 individuals were collected from industrial and semi-industrial landings in south-central Chile. The diet of T. atun was described by means of frequency, number and weight of preys, which were compared between sub-adult and adult fishes. We also measured mouth morphometry to compare them with the fish size and prey size. Mouth volume was also calculated to relate it with the number and weight of the ingested preys. Four prey species were found in their digestive tracts, Euphausia sp., Todarodes filippovae, Strangomera bentincki and Engraulis ringens, the latter being the most frequent prey $(99.9 \%)$. The mouth measures were dependent of predator body size. Equally, prey size turned out to be dependent of predator size, as well as of the mouth measures. Also, mouth volume was related positively with predator body size, and the number and the weight of the prey items were correlated with mouth size and volume. Thyrsites atun feeds in the neritic area on small fishes that live in schools. As size of T. atun increases so would increase the size of preys, and consequently when the mouth volume increases so does the quantity and biomass of prey items.
\end{abstract}

Key words: Thyrsites atun, mouth morphometrics, body size, feeding, fish schools. 


\section{INTRODUCCIÓN}

Las estrategias que desarrollan los peces para la obtención del alimento resultan determinantes en su dieta, presentando diversos hábitos de captura e ingesta de sus presas como consecuencia de sus adaptaciones morfofuncionales (Gosline 1971, Wainwrigth 1988). Son también determinantes en el tipo de presa a capturar, la ubicación, tamaño y forma de la boca y dientes (Lagler et al. 1990, Linde et al. 2004), ya que tanto el tamaño como el área de la boca se relacionan con el tamaño del pez (Karpouzi \& Stergiou 2003).

Debido a esto, en muchas especies de peces existe una estrecha relación entre la longitud del depredador y la longitud de la presa (Stergiou \& Fourtouni 1991, Villarroel \& Acuña 1999, Nilsson \& Brönmark 2000, Scharf et al. 2000, Chase 2002, Dörner \& Wagner 2003, Ibáñez et al. 2004, Ibáñez 2005) e incluso con el tamaño de la boca (Cancino \& Castilla 1985, Villarroel \& Acuña 1999, Nilsson \& Brönmark 2000, Dörner \& Wagner 2003). Además, el nivel trófico de varias especies de peces se relaciona con el área de la boca (Karpouzi \& Stergiou 2003). Entonces si el tamaño de la boca se incrementa con el crecimiento del pez, el volumen bucal también debería aumentar, lo que le permitiría al pez consumir una mayor cantidad de alimento en términos de tamaño, abundancia y biomasa, optimizando el costo energético y el tiempo de captura de las presas (Wootton 1990, Hart 1993). El volumen de la boca es un rasgo poco estudiado en los peces y podría dar cuenta de la cantidad de alimento que pueden capturar por unidad de tiempo, al abrir la boca y succionar las presas.

Las relaciones morfométricas bucales del depredador adquieren mayor importancia cuando los peces consumen presas que viven en cardúmenes. El krill, Nyctiphanes australis (Sars, 1883), cuando forma densas agregaciones en Nueva Zelanda, es consumido por cardúmenes de Thyrsites atun (Euphrasen, 1791) con mayor frecuencia que los peces solitarios (O'Driscoll 1998). En Chile, esta especie también se alimenta principalmente de animales que viven en cardúmenes, como de Eufausiáceos en zonas oceánicas (Bahamonde 1951) y de peces pequeños como la anchoveta Engraulis ringens (Jenyns, 1842), la sardina Strangomera bentincki (Norman 1936), el mote
Normanichthys crockeri Clarke 1937 y el calamar Loligo gahi, d'Orbigny, 1835, en zonas neríticas (Movillo \& Bahamonde 1971). En Sudáfrica T. atun es uno de los mayores depredadores de Engraulis capensis (Gilchrist, 1913) y Sardinops sagax (Jenyns, 1842) (Wickens et al. 1992), y se han reportado diferencias en la dieta en relación con su estado ontogénico y sexo (Griffiths 2002).

Dado que las presas pequeñas por lo general son mucho más abundantes que las presas grandes, como en el caso de las anteriormente nombradas, la mayoría de los depredadores encuentran y comen muchas más presas pequeñas que grandes (Pianka 1982). Los depredadores pequeños que comen presas pequeñas, encuentran el tamaño apropiado con mayor frecuencia que los depredadores mayores que se alimentan de presas más grandes. Como consecuencia de ello, los depredadores grandes tienden a aceptar una gama más amplia de tamaños (Pianka 1982).

El presente estudio tiene por objeto determinar variaciones en la morfometría y volumen bucal de $T$. atun y en su dieta con respecto a su tamaño corporal, como también relacionar el tamaño, número y peso de las presas que consume, con el tamaño corporal y sus medidas bucales.

\section{MATERIALES Y MÉTODOS}

Se analizó el contenido estomacal de 140 ejemplares de $T$. atun recolectados desde desembarques de naves pesqueras semiindustriales e industriales, que operan en la zona nerítica de la Octava Región, Chile centro-sur (ca. $36^{\circ} 20^{\prime} \mathrm{S}$ y $37^{\circ} 10^{\prime} \mathrm{S}$ ) (a 5 millas náuticas de la costa), entre los meses de febrero y abril del año 2004 (28 en febrero, 37 en marzo y 75 en abril).

A cada ejemplar se le midió la longitud horquilla (LH) en cm, el largo de su mandíbula inferior (LMI, cm) (Fig. 1A), abertura bucal vertical $(\mathrm{ABV}, \mathrm{cm})$, abertura bucal horizontal $(\mathrm{ABH}, \mathrm{cm})$ (Fig. 1B), y se le extrajo el estómago, los que fueron congelados a $-20{ }^{\circ} \mathrm{C}$ hasta el momento de su análisis. Posteriormente en el laboratorio, fueron analizados individualmente, donde se identificaron las presas de los contenidos estomacales al nivel taxonómico más específico posible para peces 
(Ruiz 1995) y para cefalópodos (Nesis 1987). Luego, se registró el peso de las presas (peso húmedo con $0,001 \mathrm{~g}$ de precisión) y se les midió la longitud total (LT, cm) cuando su estado de digestión lo permitió.

El análisis descriptivo y cuantitativo de los estómagos se efectuó aplicando los métodos descritos por Hyslop (1980) expresados en porcentaje: método numérico $(\% \mathrm{~N})$, método gravimétrico $(\% \mathrm{G})$ y frecuencia de ocurrencia (\% $\mathrm{F}$ ), en dos grupos de tamaños que corresponderían a los subadultos $(47-75 \mathrm{~cm}$ LH) y adultos (76-102 cm LH), ya que según Griffiths (2002), entre los 70 y 75,2 cm LH alcanzarían la primera madurez sexual a una edad de 3 años.

(A)

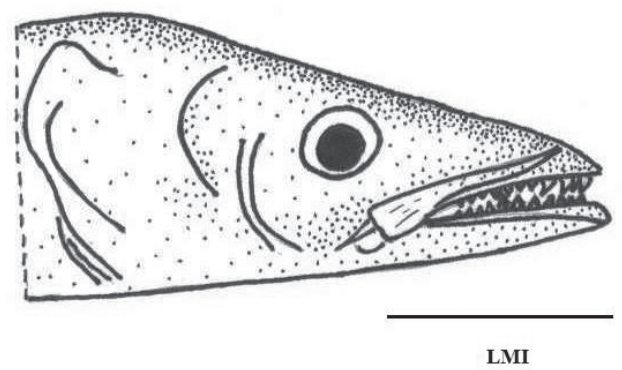

(B)

(C)
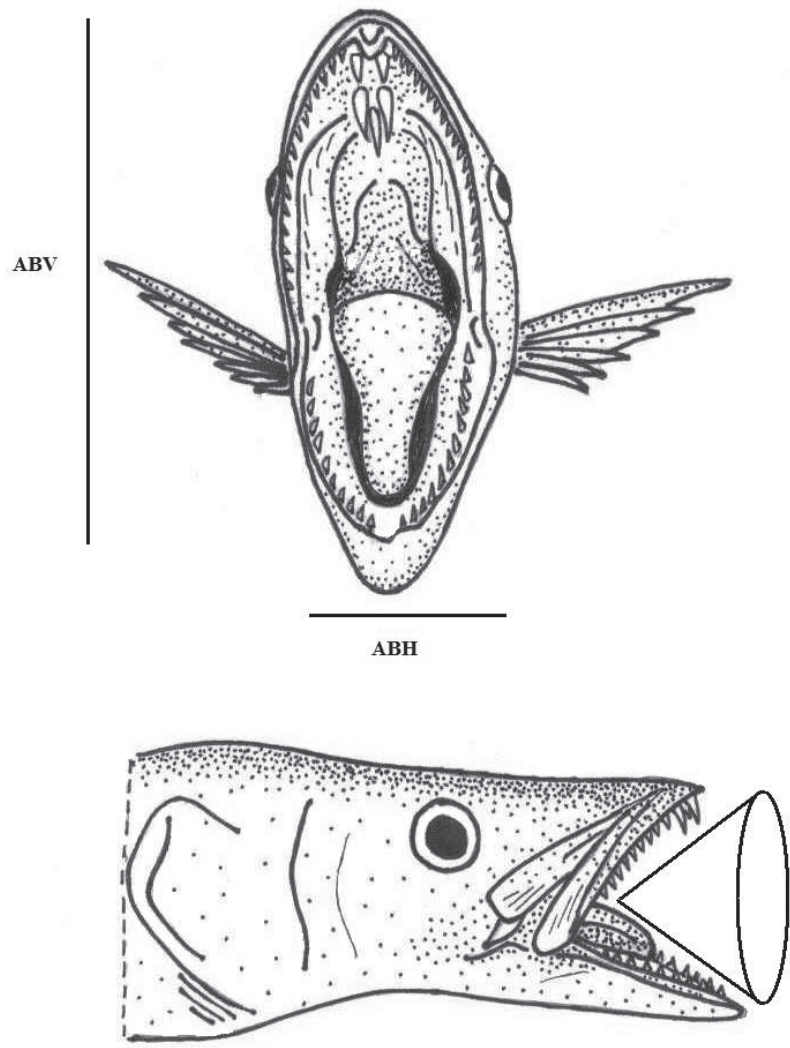

Fig. 1: Medidas bucales (cm) de T.atun. (A) LMI (longitud mandíbula inferior); (B) ABV (abertura bucal vertical) $\mathrm{ABH}$ (abertura bucal horizontal); (C) volumen bucal estimado.

Buccal measurements (cm) of T. atun. (A) LMI (lower jaw length); (B) ABV (vertical buccal aperture); ABH (horizontal buccal aperture); (C) buccal volume estimates. 
Se comparó la frecuencia de ocurrencia y el número total de cada especie presa, entre subadultos y adultos, mediante una tabla de contingencia de máxima verosimilitud y utilizando el estadígrafo de Chi-cuadrado (Zar 1984). Mientras que el peso total de cada especie presa, se comparó entre ambos grupos con la prueba no paramétrica de Friedman (Zar 1984).

Debido a que la mayoría de los peces fueron de tamaños intermedios (Fig. 2), las medidas bucales se relacionaron con el tamaño corporal de los peces para evidenciar cambios morfométricos bucales en relación con la longitud del depredador mediante ajustes lineales no paramétricos, utilizando el algoritmo RMA ("reduced major axis"). Este método minimiza los errores de ambos ejes $(x$ e $y)$, ajustándolos de acuerdo a Miller \& Kahn (1962). Se realizaron test de permutaciones para estimar la significancia del coeficiente de correlación de Pearson (r) mediante 10.000 iteraciones, y para estimar los intervalos de confianza (95\%) de la pendiente y el intercepto se utilizaron "bootstrap" con 2.000 iteraciones (Hammer et al. 2001). Igualmente, se relacionaron las medidas bucales y la longitud del depredador con el tamaño de las presas. Para establecer los ajustes entre las medidas bucales y el tamaño de los peces se utilizaron todos los individuos (con y sin contenido estomacal $n=140$ ), mientras que los ajustes lineales de las medidas morfométricas del depredador y la longitud de las presas se utilizaron solo los individuos que tenían contenido estomacal $(n=130)$ y que se le pudieron medir las presas $(n=166$ presas medidas).

Se diseñó una aproximación al cálculo del volumen de la boca, mediante el volumen de un cono, ya que el área de la boca de muchas especies de peces no es completamente circular (Karpouzi \& Stergiou 2003), y porque T. atun no es la excepción, se diseñó con base elíptica (Fig. 1C), y se describe con la siguiente expresión:

$V b=\frac{1}{3}(A B H \times A B V \times \pi) \times L M I$

Se estableció el modelo lineal que representa la asociación entre la longitud del pez y el volumen bucal estimado.

Mediante el coeficiente de correlación de Spearman (Zar 1984), se relacionó el volumen bucal $(\mathrm{Vb})$ y la longitud ( $\mathrm{LH})$ de los peces con el número y peso de las presas. En estas correlaciones la significancia fue estimada con un test de permutaciones basado en 1.000 réplicas azarosas (Hammer et al. 2001), y fueron realizadas solo con los datos de los depredadores que tenían contenido estomacal $(n=130)$.

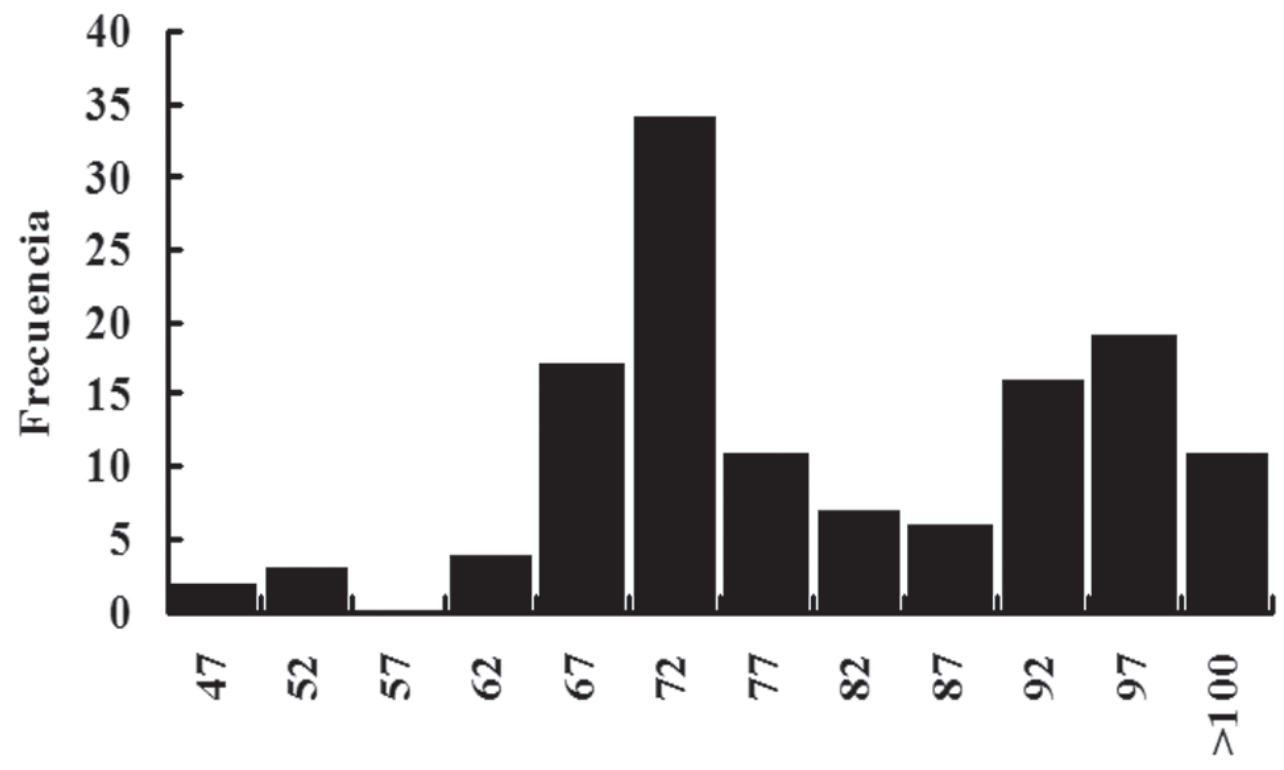

Longitud horquilla (cm)

Fig. 2: Histograma de la longitud de T. atun.

Histogram of T. atun length. 


\section{RESULTADOS}

Los ejemplares analizados presentaron tamaños que fluctuaron entre los 47 y $102 \mathrm{~cm}$ (promedio $73,8 \pm 12,1 \mathrm{DE} \mathrm{cm}$ ) de longitud horquilla (Fig. 2). Un $92,5 \%$ de los individuos analizados (130) tenía algún tipo de presa en el contenido estomacal, de los cuales la gran mayoría presentó un estado de digestión menor, lo que permitió la identificación de las presas a nivel de especie. La diversidad trófica registrada durante el período de estudio en los ejemplares subadultos, se compone solo de cuatro especies de presa: Euphausia sp., Todarodes filippovae, Strangomera bentincki y Engraulis ringens, siendo esta última la de mayor relevancia $(\mathrm{F}=$ $87 \%)$, mientras que en los ejemplares adultos se encontraron tres taxa presas, de las cuales $E$. ringens presentó la mayor frecuencia ( $\mathrm{F}=86,9$ $\%$ ) (Tabla 1). La frecuencia y número de presas no resultó dependiente de los rangos de tamaños correspondientes a subadultos $\mathrm{y}$ adultos $\left(\chi^{2}=1,66 ; \mathrm{gl}=3, \mathrm{P}=0,64 ; \chi^{2}=2,68\right.$; $\mathrm{gl}=3 ; \mathrm{P}=0,44)$, además no se encontraron diferencias en el peso de las presas (Friedman $\left.\chi^{2}=1,0 ; \mathrm{gl}=1 ; \mathrm{P}=0,31\right)$.

Los ajustes lineales realizados entre la longitud horquilla y las medidas morfométricas bucales del depredador, resultaron todos significativos (Tabla 2), e indican una relación directa entre las variables representadas, siendo
LMI la medida que se relacionó mejor con LH $(\mathrm{r}=0,87)$ (Tabla 2).

Se midieron un total de 166 presas, dos ejemplares de T. filippovae ( 11 y $18 \mathrm{~cm} \mathrm{LT}$ ), dos ejemplares de $S$. bentincki (9 y 9,5 cm LT) y 162 ejemplares de E. ringens, que presentaron tamaños que fluctuaron entre los 5 y $17 \mathrm{~cm}$ (promedio 11,2 $\pm 2,5 \mathrm{DE} \mathrm{cm}$ ) de longitud total. Los eufáusidos no se midieron debido a su estado avanzado de digestión. Como el rango de tamaño de todas las especies presas medidas fueron similares, para los análisis se consideraron como un conjunto.

La longitud de las presas resultaron dependientes del tamaño del depredador (LH) y de las medidas bucales, siendo LMI la que se relacionó mejor con la longitud de estas $(\mathrm{r}=$ 0,45) (Tabla 3) (Fig. 3, 4, 5 y 6), indicando que peces grandes tienden a consumir presas de mayor tamaño, pero debido a la gran amplitud de los datos los coeficientes de correlación no son muy altos aunque significativamente positivos. Por lo tanto, indican más bien una tendencia que muestra que a medida que los peces crecen, la magnitud de su boca aumenta y el tamaño de las presas ingeridas también.

Por otro lado, el volumen de la boca se correlacionó positivamente con la longitud de T. atun $(\mathrm{r}=0,89 ; \mathrm{P}<0,001)$, y el modelo que explica dicha relación se expresa con la siguiente ecuación: $\mathrm{Vb}=-616,43+12,225 \mathrm{LH}$.

TABLA 1

Contenido estomacal de los ejemplares subadultos y adultos de T. atun en Chile centro-sur; $\mathrm{F}=$ frecuencia de ocurrencia; $\mathrm{N}$ = número de presas; $\mathrm{G}=$ peso de las presas; $\mathrm{n}=$ número de estómagos con contenido estomacal

Stomach content of sub-adults and adults of $T$. atun from south-central Chile; $\mathrm{F}=$ frequency of occurrence; $\mathrm{N}=$ number of prey; $\mathrm{G}=$ weight of prey; $\mathrm{n}=$ number of stomachs examined

\begin{tabular}{|c|c|c|c|c|c|c|}
\hline \multirow[t]{2}{*}{ Presa } & \multicolumn{3}{|c|}{$\begin{array}{c}47-75 \mathrm{~cm} \mathrm{LH} \\
\text { Subadultos }(\mathrm{n}=69)\end{array}$} & \multicolumn{3}{|c|}{$\begin{array}{l}76-102 \mathrm{~cm} \mathrm{LH} \\
\text { Adultos }(\mathrm{n}=61)\end{array}$} \\
\hline & $\% \mathrm{~F}$ & $\% \mathrm{~N}$ & $\% \mathrm{G}$ & $\% \mathrm{~F}$ & $\% \mathrm{~N}$ & $\% \mathrm{G}$ \\
\hline \multicolumn{7}{|l|}{ Teleostei } \\
\hline Engraulis ringens & 87,0 & 93,0 & 99,4 & 86,9 & 94,4 & 99,8 \\
\hline Strangomera bentincki & 2,9 & 0,6 & 0,4 & 3,3 & 0,4 & 0,2 \\
\hline \multicolumn{7}{|l|}{ Cephalopoda } \\
\hline Todarodes filippovae & 1,4 & 0,3 & 0,2 & & & \\
\hline \multicolumn{7}{|l|}{ Euphausiacea } \\
\hline Euphausia sp. & 8,7 & 6,1 & 0,003 & 9,8 & 5,2 & 0,003 \\
\hline Total & & 330 & $3.162,2$ & & 484 & $7.766,7$ \\
\hline
\end{tabular}


TABLA 2

Ajustes lineales entre la longitud horquilla y las mediciones morfométricas bucales de T. atun en Chile centro-sur $(\mathrm{n}=140$ depredadores medidos $)$ : $\mathrm{MI}=$ longitud mandíbula inferior; $\mathrm{ABH}=$ abertura bucal horizontal; $\mathrm{ABV}=$ abertura bucal vertical

Linear fits between fork length and mouth morphometric measurements of T. atun from south-central Chile $(\mathrm{n}=140$ measured predators); $\mathrm{LMI}=$ lower jaw length; $\mathrm{ABH}=$ horizontal buccal aperture; $\mathrm{ABV}=$ vertical buccal aperture

\begin{tabular}{lcccccc}
\hline Medida & Valor de a & Valor de $\mathrm{b}$ & Valor de $\mathrm{r}$ & Valor de P & $\begin{array}{c}\text { Intervalo confianza } \\
95 \% \text { de a }\end{array}$ & $\begin{array}{c}\text { Intervalo confianza } \\
95 \% \text { de } \mathrm{b}\end{array}$ \\
\hline LMI & 1,55 & 0,08 & 0,87 & $<0,001$ & $0,85-2,25$ & $0,07-0,09$ \\
ABH & $-1,29$ & 0,08 & 0,53 & $<0,001$ & $-2,53--0,44$ & $0,07-0,10$ \\
ABV & $-2,84$ & 0,13 & 0,48 & $<0,001$ & $-4,46--1,72$ & $0,11-0,15$ \\
\hline
\end{tabular}

TABLA 3

Ajustes lineales entre las variables bucales y la longitud horquilla y la longitud de las presas y de $T$. atun en Chile centro-sur ( $\mathrm{n}=166$ presas medidas); $\mathrm{LH}=$ longitud horquilla; $\mathrm{LMI}=$ longitud mandíbula inferior; $\mathrm{ABH}=$ abertura bucal horizontal; $\mathrm{ABV}=$ abertura bucal vertical

Linear fits between mouth morphometric measurements and fork length and prey length of $T$. atun from south-central Chile ( $\mathrm{n}=166$ measured prey); $\mathrm{LH}=$ fork length; $\mathrm{LMI}=$ lower jaw length; $\mathrm{ABH}=$ horizontal Buccal opening; $\mathrm{ABV}=\mathrm{vertical}$ Buccal opening

\begin{tabular}{lcccccc}
\hline Medida & Valor de a & Valor de $\mathrm{b}$ & Valor de $\mathrm{r}$ & Valor de P & $\begin{array}{c}\text { Intervalo confinza } \\
95 \% \text { de a }\end{array}$ & $\begin{array}{c}\text { Intervalo confinza } \\
95 \% \text { de } b\end{array}$ \\
\hline LH & $-17,43$ & 0,40 & 0,44 & $<0,001$ & $-24,35--12,95$ & $0,34-0,50$ \\
LMI & $-24,50$ & 4,95 & 0,45 & $<0,001$ & $-31,25--19,9$ & $4,29-5,90$ \\
ABH & $-11,36$ & 4,69 & 0,23 & $<0,001$ & $-15,22--8,34$ & $4,06-5,49$ \\
ABV & $-8,53$ & 3,12 & 0,27 & $<0,001$ & $-11,3--5,95$ & $2,72-3,54$ \\
\hline
\end{tabular}

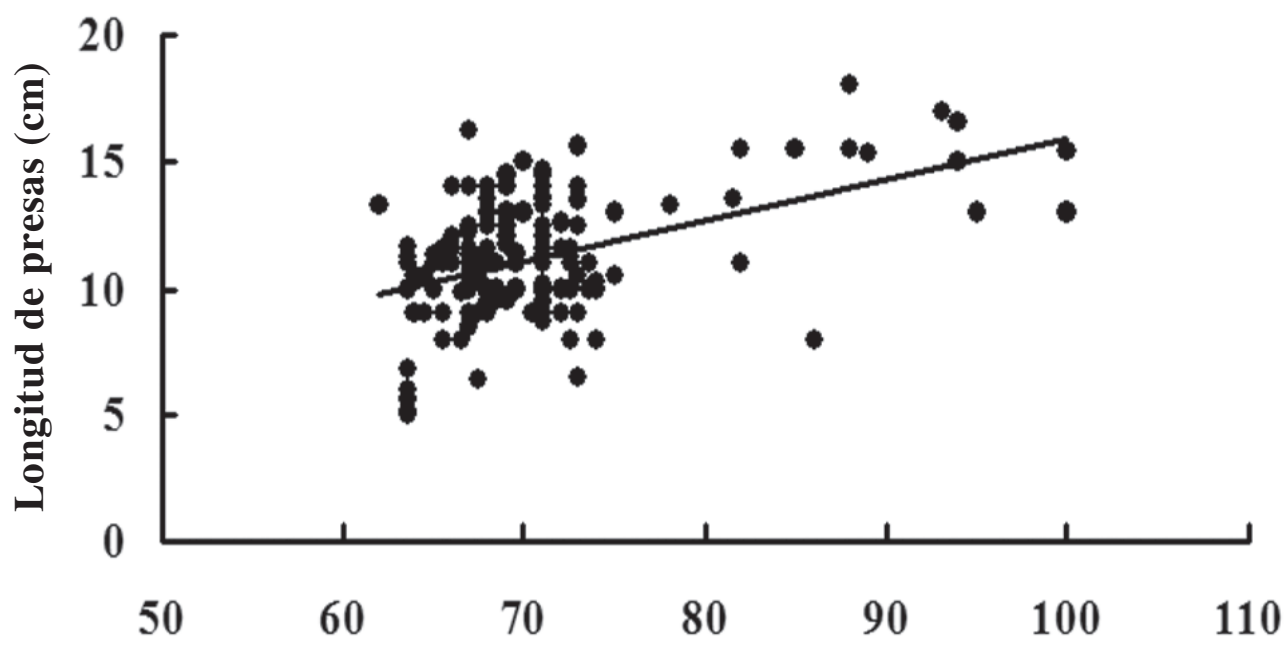

\section{Longitud horquilla (cm)}

Fig. 3: Relación entre la longitud horquilla $(\mathrm{cm})$ de $T$. atun y la longitud de presas $(\mathrm{cm})$. Fork length (cm) of T. atun and prey length (cm) relationship. 


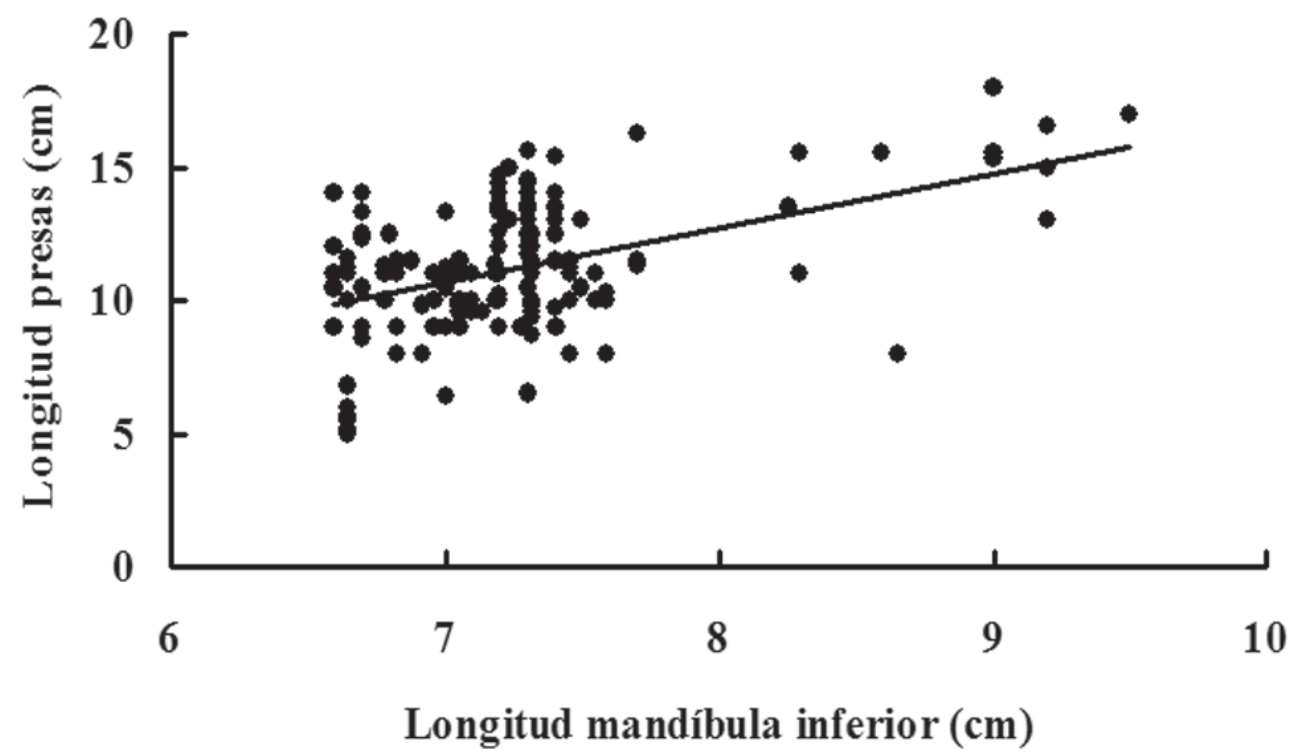

Fig. 4: Relación entre el largo de la mandiíbula inferior $(\mathrm{cm})$ y longitud de presas $(\mathrm{cm})$ de T. atun. Lower jaw length $(\mathrm{cm})$ of $T$. atun and prey length $(\mathrm{cm})$ relationship.

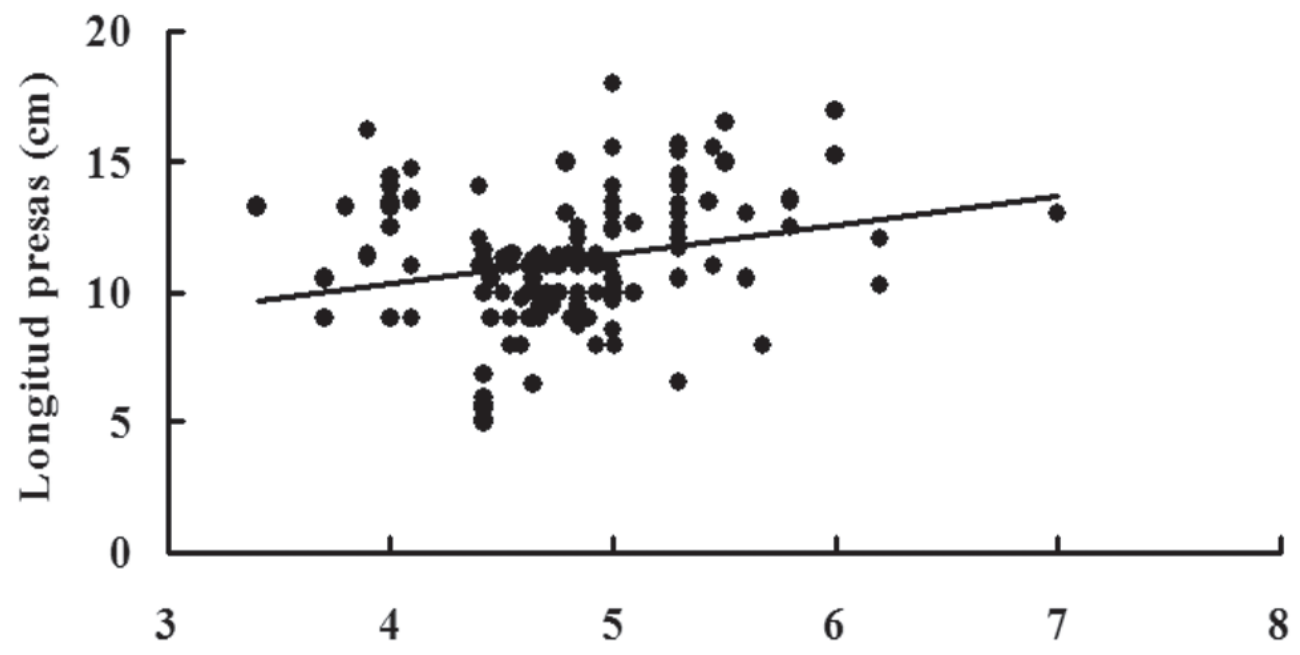

Abertura bucal horizontal (cm)

Fig. 5: Relación entre la abertura bucal horizontal $(\mathrm{cm})$ y longitud de presas $(\mathrm{cm})$ de $T$. atun. Horizontal buccal aperture $(\mathrm{cm})$ of $T$. atun and prey length $(\mathrm{cm})$ relationship. 


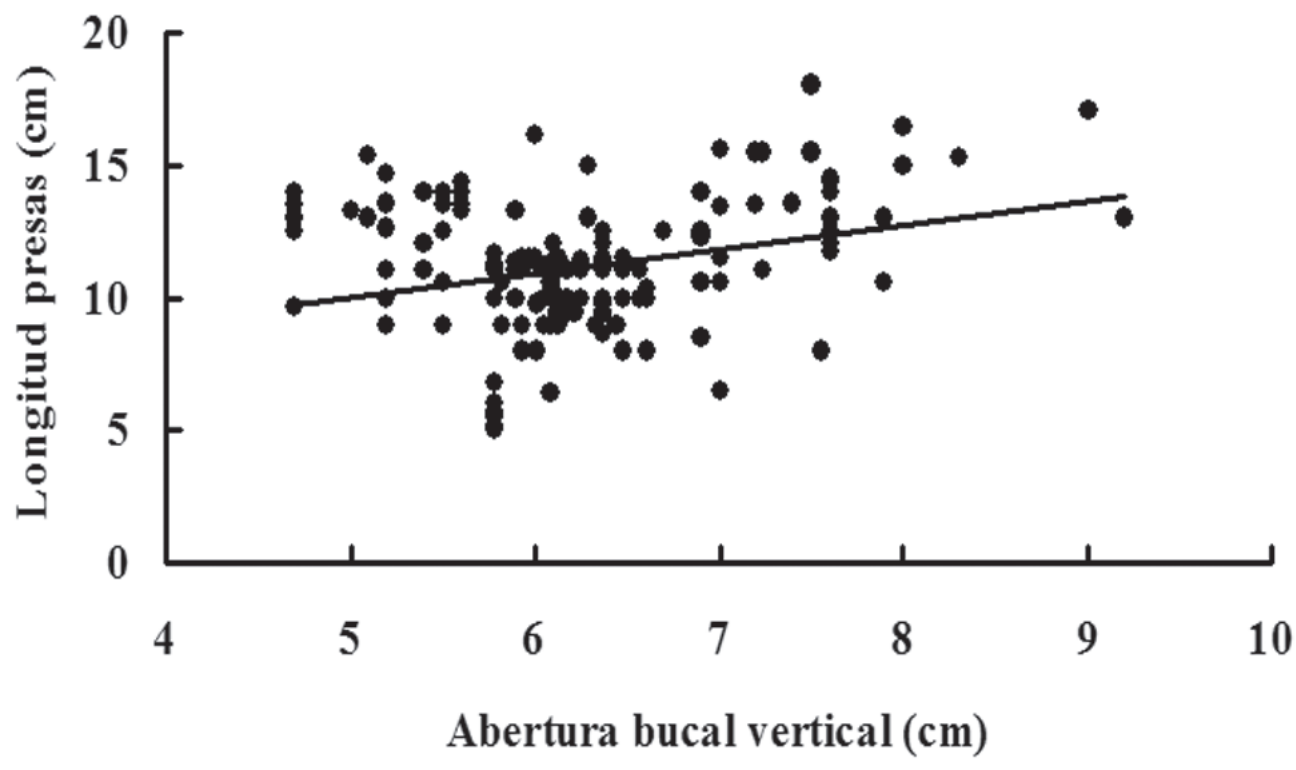

Fig. 6: Relación entre la abertura bucal vertical $(\mathrm{cm})$ y longitud de presas $(\mathrm{cm})$ de T. atun. Vertical buccal aperture $(\mathrm{cm})$ of $T$. atun and prey length $(\mathrm{cm})$ relationship.

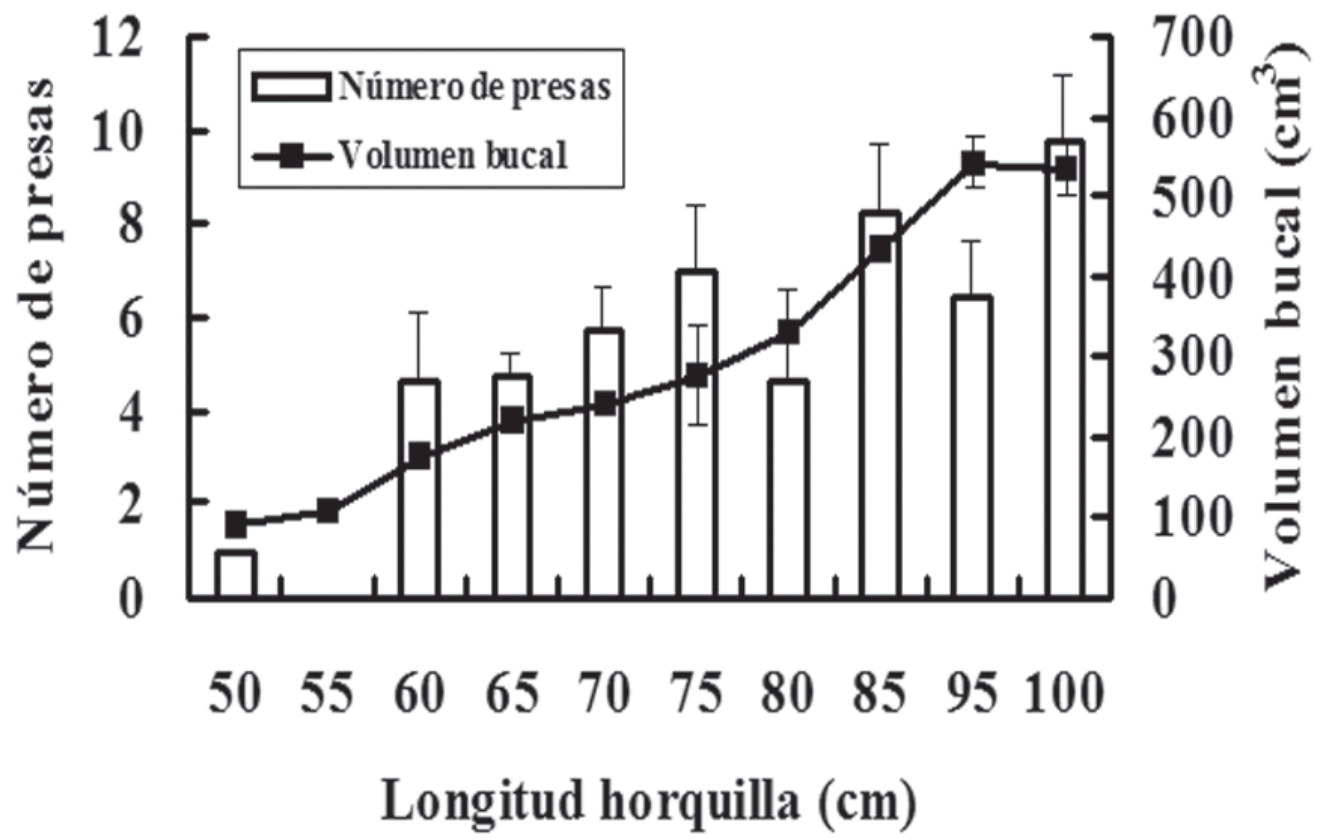

Fig. 7: Número de presas y volumen bucal $\left(\mathrm{cm}^{3}\right)$ en relación con la longitud de T. atun (cm). Las barras representan el error estándar.

Prey number and buccal volume $\left(\mathrm{cm}^{3}\right)$ related to $T$. atun length $(\mathrm{cm})$. Bars represent standard errors. 

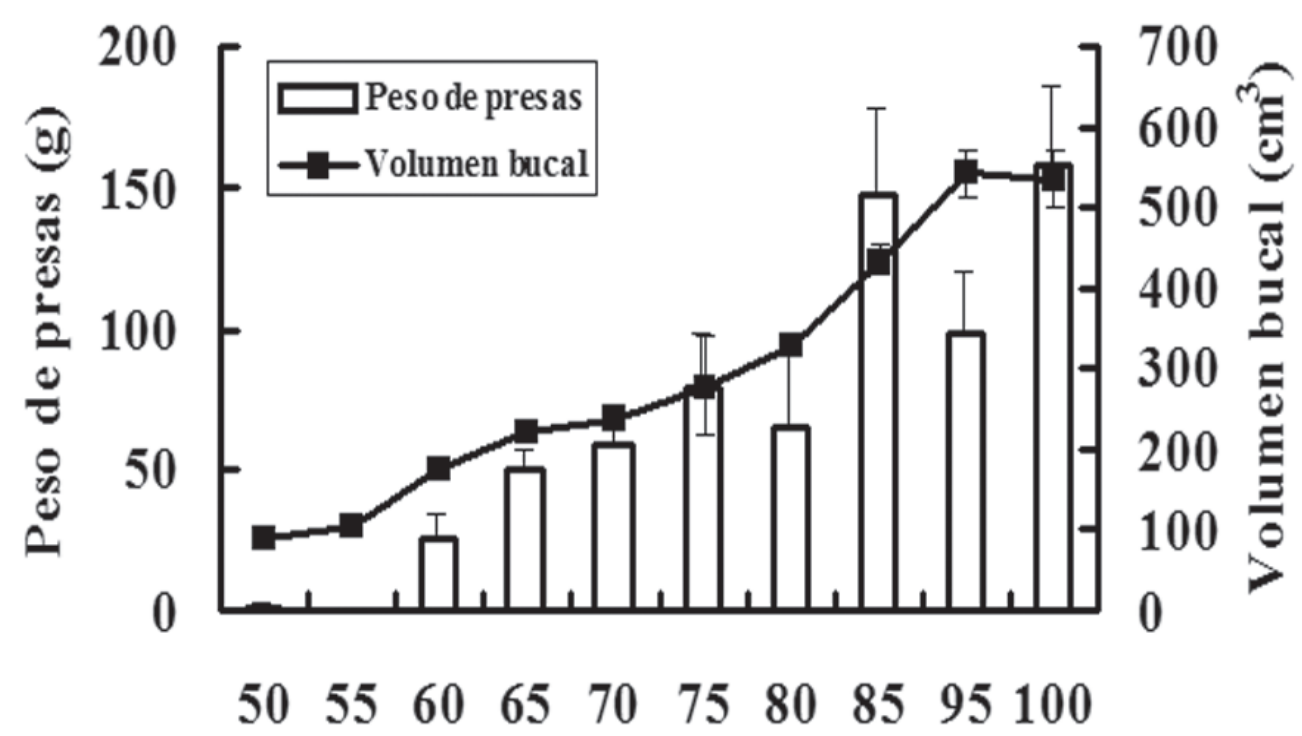

\section{Longitud horquilla (cm)}

Fig. 8: Peso de presas (g) y volumen bucal $\left(\mathrm{cm}^{3}\right)$ en relación con la longitud de T. atun (cm). Las barras representan el error estándar.

Prey weight $(\mathrm{g})$ and buccal volume $\left(\mathrm{cm}^{3}\right)$ related to $T$. atun length $(\mathrm{cm})$. Bars represent standard errors

Asimismo el número (Fig. 7) y peso (Fig. 8) de las presas se correlacionaron con la longitud de los peces $\left(\mathrm{r}_{\mathrm{s}}=0,39 ; \mathrm{n}=130 ; \mathrm{P}<0,001 ; \mathrm{r}_{\mathrm{s}}=\right.$ 0,$49 ; \mathrm{n}=130 ; \mathrm{P}<0,001$, respectivamente), como con el volumen de la boca $\left(\mathrm{r}_{\mathrm{s}}=0,38 ; \mathrm{n}=\right.$ $130 ; \mathrm{P}<0,001 ; \mathrm{r}_{\mathrm{s}}=0,45 ; \mathrm{n}=130 ; \mathrm{P}<0,001$, respectivamente).

\section{DISCUSIÓN}

Durante el período de estudio, la dieta de $T$. atun consistió básicamente en E. ringens, y otras tres especies con una baja importancia, lo cual indica que posee un reducido espectro trófico. Según un estudio anterior, realizado en el norte del archipiélago de Chiloé por Bahamonde en (1951), la dieta de T. atun es muy distinta a la de este estudio, donde las presas más importantes fueron los Euphausiáceos $(63,93 \%)$ y Teleósteos $(26,54$ $\%)$. Todas estas especies viven en cardúmenes, y considerando que este depredador es un rápido nadador que posee una poderosa dentadura, puede capturar un gran número y biomasa de presas relativas a su tamaño, al embestirse contra cardúmenes de peces, eufáusidos y cefalópodos, su principal alimento en las zonas neríticas de Chile centro-sur.

Además, T. atun forma pequeñas agregaciones en las zonas neríticas de Chile centro-sur, y considerando que no se han encontrado ejemplares sexualmente maduros en un periodo anual, estas áreas serían exclusivamente de alimentación, en cambio en aguas oceánicas, es más probable encontrarlo en solitario y en algunas ocasiones maduros ( $\mathrm{J}$. Chong, datos no publicados).

No se encontraron variaciones dietarias con respecto a las categorías de tamaños (subadultos y adultos) de T. atun en cuanto a número, peso y frecuencia de las presas. Estos resultados no son significativos, puesto que no se incluyeron ejemplares juveniles, los cuales se alimentan mayoritariamente de anfípodos, eufáusidos y mictófidos en otras latitudes (Griffiths 2002). En la zona de estudio no se encontraron ejemplares juveniles $(<40 \mathrm{~cm}$ LH), los que podrían alimentarse en aguas oceánicas, y considerando que cambios ontogénicos en la dieta estarían asociados con diferencias en el hábitat (Stergiou \& Fortouni 1991), si los ejemplares juveniles se encuentran en otra zona, estarían alimentándose 
principalmente de pequeños crustáceos planctónicos.

La mayoría de los peces medidos, y consecuentemente también las presas, fueron de tamaños intermedios (Fig. 2) influenciando los resultados de las correlaciones, las cuales no tuvieron un ajuste superior a un $r=0,45$. No obstante se encuentra una tendencia a aumentar el tamaño de las presas en relación a la longitud del depredador y de su mandíbula inferior. Tanto el tamaño como el volumen de la boca se relacionaron positivamente con el tamaño corporal en $T$. atun, implicando que individuos pequeños están limitados en el tamaño, número y volumen de presas que podrían capturar. También se podría hipotetizar que los peces más grandes nadarían a una mayor velocidad y por lo tanto, podrían capturar presas de mayor tamaño que los peces pequeños que no serían capaces de alcanzar presas de mayor longitud.

A diferencia de los depredadores pequeños, los más grandes pueden comer un amplio rango de tamaños de presas (Cohen et al. 1993). De hecho, puede constituir una mejor estrategia para un depredador grande dejar de lado presas inferiores y dedicar el tiempo que gastaría en capturar e ingerir presas pequeñas, a buscar presas más grandes (Pianka 1982). Por otro lado, el tamaño de las presas relativo al tamaño del depredador, puede decrecer significativamente al aumentar el tamaño del pez (Dörner \& Wagner 2003). Sin embargo, el tamaño de las presas en relación con el tamaño corporal de $T$. atun no cambia, manteniéndose alrededor del $20 \%$ de la longitud de $T$. atun, pero debido a los pocos datos de depredadores grandes $(>80 \mathrm{~cm} \mathrm{LH})$, encontramos la mayor variación en el tamaño de las presas en los ejemplares entre 62 y $74 \mathrm{~cm}$ $\mathrm{LH}$, lo que influencia fuertemente los ajustes lineales (coeficientes de correlación e intervalos de confianza de los interceptos y pendientes, Tabla 3).

Las morfometrías bucales y el tamaño de $T$. atun presentaron una tendencia creciente con el tamaño de las presas consumidas, ya que los peces con morfometrías bucales y tamaños mayores consumen presas más grandes. Estudios en otras especies de peces indican que a medida que el tamaño corporal aumenta, también lo hace el tamaño de presas encontradas en sus estómagos (Stergiou \& Fourtouni 1991, Villarroel \& Acuña 1999, Scharf et al. 2000, Chase 2002, Dörner \&
Wagner 2003, Ibáñez et al. 2004, Ibáñez 2005). Incluso esta relación es positiva en un gran número de depredadores en ambientes terrestres, acuáticos, costeros y marinos (Cohen et al. 1993). Esto, en parte, debido a que el tamaño corporal es una de las características más importantes de los animales (SchmidtNielsen 1984), ya que influye en cuanta energía necesitan y cuanto alimento pueden capturar, entre otros aspectos de su historia de vida (Cohen et al. 1993). El volumen de la boca podría ser un buen estimador de la capacidad de alimento que pueden capturar los peces, ya que se correlaciona con el número y biomasa de presas encontradas en el estómago de los depredadores, pero para mejorar los ajustes (eventualmente) se deben medir un mayor número de peces en cada rango de longitud para confirmar la variabilidad del tamaño de las presas ingeridas.

Finalmente, evidenciamos una gran presión depredatoria de $T$. atun sobre ejemplares sexualmente maduros ( $>10 \mathrm{~cm} \mathrm{LT}$ ) de $E$. ringens (Cubillos et al. 1999), y al ser la presa más importante dentro del espectro trófico de $T$. atun en las zonas neríticas en Chile centrosur, pensamos que esta presión provocaría un impacto en la dinámica poblacional de esta especie, como lo registrado en Sudáfrica por el mismo depredador (Wickens et al. 1992). La depredación por peces piscívoros es la mayor causa de mortalidad de poblaciones de peces de agua dulce, y puede afectar la densidad además de la estructura poblacional de las presas (Brönmark et al. 1995, Nilsson \& Brönmark 2000, Dörner \& Wagner 2003). Sin embargo, las presas evitan la depredación mediante diversos mecanismos, y considerando lo numerosos que son los cardúmenes de $E$. ringens, es muy probable que utilicen estrategias como la confusión del depredador y el efecto de dilución (Pitcher \& Parrish 1993).

\section{LITERATURA CITADA}

BAHAMONDE N (1951) Alimentación de la sierra (Thyrsites atun Euphrasen 1791). Investigaciones Zoológicas Chilenas (Chile) 1: 8-10.

BRÖNMARK C, CA PASZKOWSKI, WM TONN \& A HARGEBY (1995) Predation as a determinant of size structure in populations of crucian carp (Carassius carassius) and tench (Tinca tinca). Ecology of Freshwater Fish 4: 85-92.

CANCINO J \& J CASTILLA (1985) Emersion behaviour and 
foraging ecology of the common Chilean clingfish Sicyases sanguineus (Pisces: Gobiesocidae). Journal of Natural History 22: 249-261

CUBILLOS L, M CANALES, D BUCAREY, A ROJAS \& R ALARCÓN (1999) Época reproductiva y talla media de primera madurez sexual de Strangomera bentincki y Engraulis ringens en el período 19931997, en la zona centro-sur de Chile. Investigaciones Marinas (Chile) 27: 73-85.

CHASE BC (2002) Differences in diet of Atlantic bluefin tuna (Thunnus thynnus) at five seasonal feeding grounds on the New England continental shelf. Fishery Bulletin 100: 168-180.

COHEN JE, SL PIMM, P YODZIS \& J SALDAÑA (1993) Body sizes of animal predators and animal prey in food webs. Journal of Animal Ecology 62: 67-78.

DÖRNER H \& A WAGNER (2003) Size-dependent predator-prey relationships between perch and their fish prey. Journal of Fish Biology 62: 1021-1032.

GOSLINE W (1971) Functional morphology and classification of teleostean fishes. The University Press of Hawai, Honolulu, USA. 208 pp.

GRIFFITHS MH (2002) Life history of South African snoek, Thyrsites atun (Pisces: Gempylidae): a pelagic predator of the Benguela ecosystem. Fishery Bulletin 100: 690-710.

HAMMER $\varnothing$, DAT HARPER \& PD RYAN (2001) PAST: Paleontological statistics software package for education and data analysis. Palaeontologia Electronica 4: 1-9.

HART PJB (1993) Teleost foraging: facts and theories. En: Pitcher TJ (ed) Behaviour of teleost fishes: 253362. Second edition. Chapman \& Hall, London, United Kingdom.

HYSLOP E (1980) Stomach content analysis: a review of methods and their application. Journal of Fish Biology 17: 411-429.

IBÁÑEZ CM, C GONZÁLEZ \& L CUBILLOS (2004) Dieta del pez espada Xiphias gladius Linnaeus, 1758, en aguas oceánicas de Chile central en invierno de 2003. Investigaciones Marinas (Chile) 32: $113-120$.

IBÁÑEZ CM (2005) Relaciones morfométricas del draco rayado Champsocephalus gunnari (Perciformes, Notothenioidei) y su presa el krill antártico Euphausia superba (Crustacea, Euphausiacea). Boletín del Museo Nacional de Historia Natural (Chile) 54: 33-36.

LINDE M, M PALMER \& J GÓMEZ-ZURITA (2004) Differential correlates of diet and phylogeny on the shape of the premaxila and anterior tooth in sparid fishes (Perciformes: Sparidae). Journal of Evolutionary Biology 17: 941-952.

MILLER RL \& JS KAHN (1962) Statistical analysis in the geological sciences. John Wiley \& Sons, New York, New York, USA. 483 pp.

MOVILLO J \& N BAHAMONDE (1971) Contenido gástrico y relaciones tróficas de Thyrsites atun (Euphrasen) en San Antonio, Chile. Boletín del
Museo Nacional de Historia Natural (Chile) 29: 289-338.

NESIS K (1987) Cephalopods of the world. T.F.H. Publications Inc., city, New Jersey, USA. $351 \mathrm{pp}$.

NILSSON PA \& C BRÖNMARK (2000) Prey vulnerability to a gape-size limited predator: behavioural and morphological impacts on northern pike piscivory. Oikos 88: 539-546.

O'DRISCOLL RL (1998) Feeding and schooling behaviour of barracouta (Thyrsites atun) off Otago. New Zealand Marine and Freshwater Research 49:19-24.

PIANKA ER (1982) Ecología evolutiva. Segunda edición. Editorial Omega, Barcelona, España. 363 pp.

PITCHER TJ \& JK PARRISH (1993) Functions of shoaling behaviour in teleosts. En: Pitcher TJ (ed) Behaviour of teleost fishes: 364-439. Second edition. Chapman \& Hall, London, United Kingdom.

KARPOUZI VS \& KI STERGIOU (2003) Mouth shape and relationships between mouth size and body length for several marine fishes: trophic implications. Journal of Fish Biology 62: 13531365.

RUIZ VH (1995) Catálogo de los peces marinos comunes de la Octava Región. Editorial de la Universidad de Concepción, Concepción, Chile. 226 pp.

SCHARF FS, F JUANES \& RA ROUNTREE (2000) Predator size-prey size relationships of marine fish predators: interspecific variation and effects of ontogeny and body size on trophic-niche breadth Marine Ecology Progress Series 208: 229-248.

SCHMIDT-NIELSEN K (1984) Scaling: Why is animal size so important? Cambridge University Press, Cambridge, United Kingdom. 617 pp.

STERGIOU KI \& H FOURTOUNI (1991) Food habits, ontogenetic diet shift and selectivity in Zeus faber Linnaeus, 1758. Journal of Fish Biology 39: 589603.

VILLARROEL JC \& E ACUÑA (1999) Alimentación y relaciones predador-presa en el lenguado de ojos grandes Hippoglossina macrops Steindachner 1876 (Pisces: Paralichthyidae) de la zona norte de Chile. Revista de Biología Marina y Oceanografía (Chile) 34: 145-154.

WAINWRIGTH PC (1988) Morphology and ecology: functional basis of feeding constraints in Caribbean labrid fishes. Ecology 69: 635-645.

WICKENS P, D JAPP, P SHELTON, F KRIEL, P GOOSEN, B ROSE, C AUGUSTYN, C BROSS, A PENNEY \& R KROHN (1992) Seals and fisheries in South Africa - Competition and conflict. South African Journal of Marine Science 12: 773-789.

WOOTTON RJ (1990) Ecology of teleost fishes. Chapman and Hall, London, United Kingdom. 404 pp.

ZAR JH (1984) Biostatistical analysis. Second edition. Prentice-Hall Inc., Englewood Cliffs, New Jersey, USA. 662 pp. 
